\title{
Perceptual learning of orientation judgments in oblique meridians
}

\author{
Gerald Westheimer • Jonathan Lavian
}

Published online: 25 May 2013

(C) Psychonomic Society, Inc. 2013

\begin{abstract}
Fourteen daily training sessions in orientation discrimination of foveal lines in the 45-deg meridian improved thresholds in the trained meridian by an average of $25 \%$ in five observers. A substantial amount of training transferred to the other obliques, but none to the cardinal meridians, with a consequent reduction in the oblique effect. The data were interpreted as showing perceptual learning at two levels: performance facilitation specific to the trained orientation and improved proficiency globally. The failure of the cardinal orientations to share in the benefit is likely to have its origin in the fact that contour orientation in these meridians is so well established that it had already reached maximum hyperacuity thresholds. The judgment of obliques depends much more than the judgment of cardinals on whether the comparison and test stimuli are shown simultaneously or in succession, but this effect is not changed by perceptual training.
\end{abstract}

Keywords Oblique effect · Meridional anisotropia ·

Perceptual learning $\cdot$ Short-term memory

Since Mach first observed that the orientations of vertical and horizontal lines can be judged better than those of oblique lines, the oblique effect, as it has since been called (Appelle, 1972), has been widely demonstrated, yet it has resisted a unitary explanation. The small predominance of neurons in the visual cortex that are tuned to cardinal orientations in the alert primate (Zhang et al., 2010) does not suffice as an explanation, because some very elementary visual tasks do not manifest deficits in oblique meridians,

G. Westheimer · J. Lavian

Division of Neurobiology, University of California,

Berkeley, CA, USA

G. Westheimer $(\square)$

144 Life Sciences Addition, University of California,

Berkeley, CA, USA

e-mail: gwestheimer@berkeley.edu whereas others, whose principal neural substrates are obviously more central and distributed than the primary visual cortex, do (Westheimer, 2003).

Though orientation-specific perceptual learning had been the subject of an earlier inquiry in connection with vernier acuity (McKee \& Westheimer, 1978), perceptual learning in the task of discriminating line orientation was first demonstrated by Vogels and Orban (1985), who found evidence for such learning from stimuli with an oblique orientation, but not from those with a cardinal orientation. Improvement through training in an oblique orientation was affirmed by Shiu and Pashler (1992), but their evidence for lack of transfer pertained only to retinal location, not to orientation. A detailed study by Schoups, Vogels, and Orban. (1995) failed to reveal any transfer of learning to other orientations or retinal locations; this, together with binocular transfer, hints strongly that the involved circuits are restricted to the primary visual cortex, though evidence of changes of tuning curves in V4 of monkeys suggests the involvement of higher visual areas (Raiguel, Vogels, Mysore, \& Orban, 2006). Recently, Tschopp-Junker, Gentaz, and Viviani (2010) provided further evidence that performance in oblique meridians improves through training, but not enough to eradicate the oblique effect. Though all of these studies involved several thousand responses with feedback, spread over several days and using high-contrast stimuli, the test and training procedures differed among the studies.

The conceptual importance for the oblique effect of transfer of training across orientations motivated us to examine the question of whether substantial training of orientation judgments in a single oblique meridian would result in a performance improvement, and if so, whether the improvement would be specific to the trained meridian or attributable to generalized perceptual learning. Training five observers in one meridian, with detailed pre- and posttraining characterization of their orientation discrimination in eight orientations, enabled us to reach answers to these questions. In the process, we were able to shed some light on the difference in the 
oblique effect between simultaneous and successive exposures of comparison and test stimuli (Heeley \& BuchananSmith, 1992).

\section{Method}

Procedure

Observers had to detect the direction of misorientation of a foveal line presented for $250 \mathrm{~ms}$, using a pair of flanking lines as a comparison (Fig. 1a). During each run of 150 or more trials, which occurred at regular 3-s intervals and were restricted to one meridian, the line was shown at random in one of seven orientations that ranged from 3.6 deg counterclockwise to 3.6 deg clockwise with respect to the two flanking lines, which remained at the standard orientation throughout each run. The flanking lines were located $0.5 \mathrm{deg}$ to each side of the central line. After each presentation, observers responded with a mouse buttonpress to judge whether they perceived the test line as being tilted counterclockwise or clockwise with respect to the flanking lines. Error feedback was provided by an auditory signal. In the main experiment, the flankers were shown (Fig. 1b) in four different temporal relationships with respect to the test line: either synchronous or exposed for $250 \mathrm{~ms}$ with an onset preceding the test line's onset by 250,500 , or $750 \mathrm{~ms}$. In an additional, subsidiary experiment (see Fig. 6 below), the comparison was not a flanking pair of lines but a large square that was shown either continuously or with a temporal gap created by a 650- or 250-ms onset asynchrony.

\section{Observers}

The observers were undergraduate biology students, four male and one female, about age 20, with unexceptional optometric status and no previous experience in this kind of experiment, except for one or two brief introductory runs in related visual tasks. The experiments were approved by the Institutional Review Board and were in conformity with the principles of the Helsinki Declaration.

\section{Stimuli}

The stimuli were generated on laptop computers on liquid crystal display (LCD) screens, using a central gray disk, 400 pixels in diameter, as a fixation area and background. The line stimuli were white, with a Michelson contrast of about 0.4 , and were 2 arcmin wide and 48 arcmin in length. The refresh rate of $60 \mathrm{~Hz}$ and the light extinction typical of modern LCDs assured that the timing program of $250 \mathrm{~ms}$ exposure was adequately adhered to. Observation was a

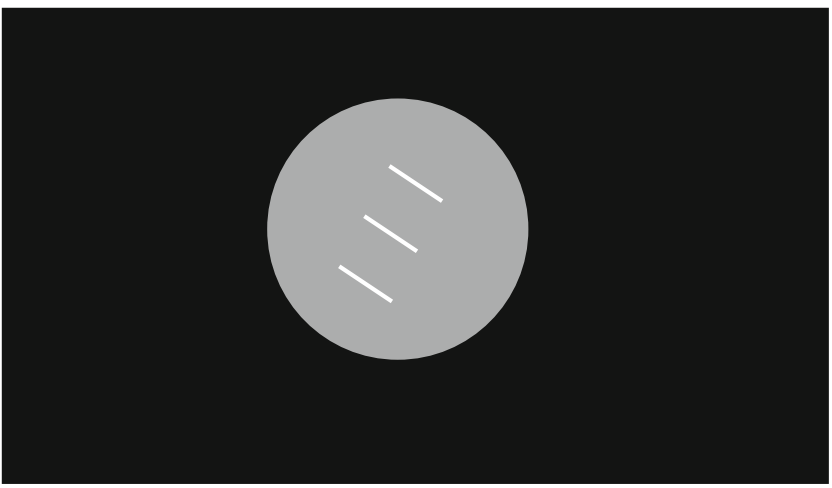

b

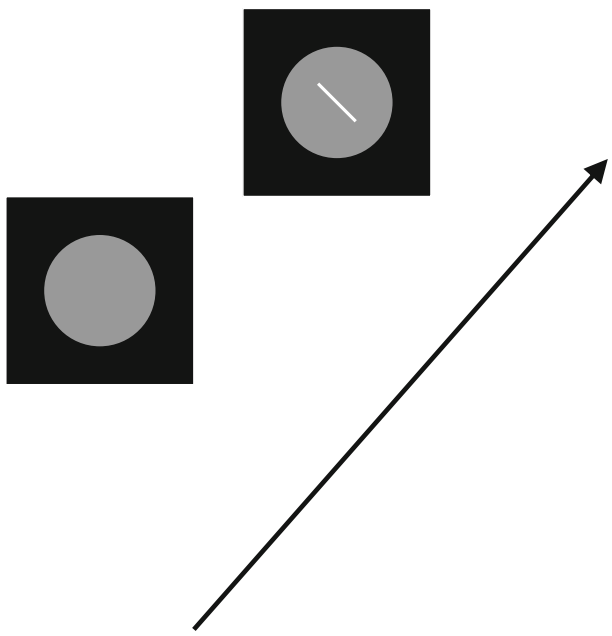

Fig. 1 (a) Observer's view of the experimental configuration of a gray disk, on which were superimposed the central test line, 48 arcmin in length and exposed for $250 \mathrm{~ms}$, and its two flanking lines, $0.5 \mathrm{deg}$ to each side and incorporating the standard orientation. The three-line pattern could be shown in eight orientations spaced $22.5 \mathrm{deg}$ apart. The temporal relationships of these stimuli are illustrated in panel $b$. The gray disk was seen in the center of the (otherwise black) laptop screen and typically subtended $15 \times 12 \mathrm{deg}$ at the observer's eyes. (b) Time line illustrating the delay situation when the flankers were exposed first for $250 \mathrm{~ms}$, followed by a pause that lasted 0,250 , or $500 \mathrm{~ms}$, and then by presentation of the test line for $250 \mathrm{~ms}$ at an orientation that was perturbed with respect to the standard embodied by the flankers. Observers had to register whether their perceived orientation of the test line was inclined counter- or clockwise compared to the standard, which remained constant throughout a run of 600 trials, at 3-s intervals, in which simultaneity of test and standard, and the three delays were randomly intermixed

binocular at distances, typically 70 and $90 \mathrm{~cm}$, such that each display pixel subtended 1 arcmin at the eye.

The program allowed the patterns to be presented in eight orientations, $22.5 \mathrm{deg}$ apart around the face of a virtual clock, and care was exercised to ensure that all lines, which were generated with an antialiasing algorithm, fitted in with the square screen raster. This necessitated small adjustments, depending on stimulus orientation, in the magnitude of the perturbations by which the probing stimuli differed from the standard. 
Data acquisition sequence

The sequence of events for each observer was as follows.

(1) For the pretraining baseline, two runs, on separate days, of 600 trials each, were performed for each of the eight orientations, with 150 trials for each asynchrony value, yielding a baseline orientation discrimination threshold. Each run was devoted to just one orientation, but in the manner of the constant-stimulus psychophysical procedure, the test line was shown at random in one of the seven perturbations with the four asynchronies, which were randomly interspersed.

(2) This was followed by 14 days of training, spread over $\sim 3$ weeks, with 600 responses per day, using only the 45-deg oblique orientation and all four asynchronies randomly interspersed.

(3) For posttraining, the sequence of baseline runs was repeated.
Data analysis

By means of a probit fit, an orientation discrimination threshold, with a standard error, is extracted for each condition in each run. This is a measure of the orientation difference whose direction of deviation (clockwise or counterclockwise) from the standard can be correctly identified $75 \%$ of the time.

For the pre- and posttraining data, results from the two runs were summed, and the threshold and its standard error computed from the summed data. To reach overall conclusions, the data from all five observers were averaged. The error of the means of each average threshold value was computed by finding the root-mean square of the constituent variances, and this was then used for paired $t$ tests to assign significance to the pre- and posttraining differences.
Fig. 2 Averaged orientation discrimination thresholds for all five observers, for each of the eight orientations and four onset asynchronies of the comparison, before and after 14 days of training on the 45 deg meridian (marked by the arrows)
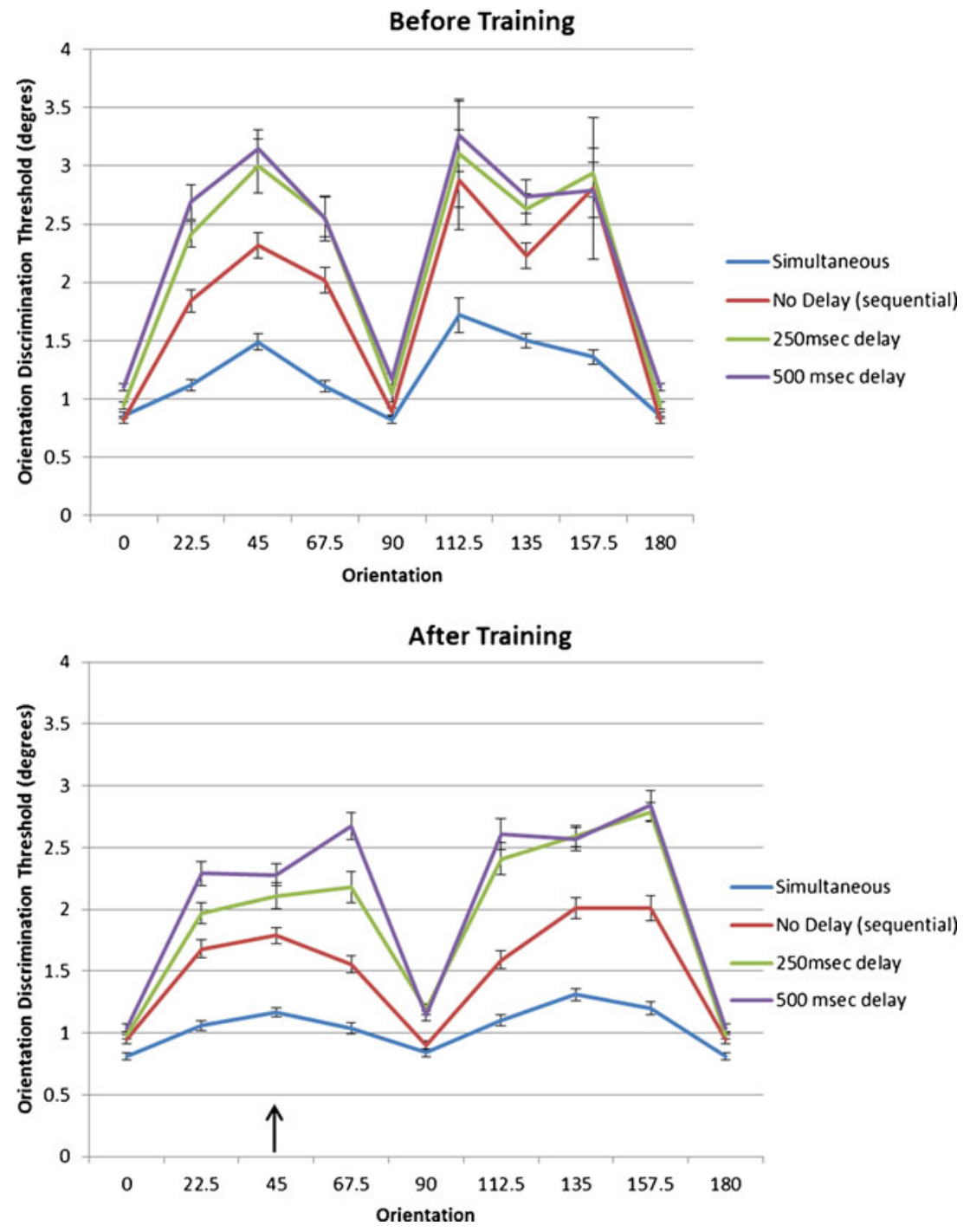
Fig. 3 Summary of the line orientation discrimination data in terms of degrees (Fig. 2), showing perceptual learning of orientation discrimination in the trained meridians (45 deg), partial transfer to other obliques, and a lack of transfer to the cardinal meridians. Preand posttraining differences are significant for the oblique $(p s<.01)$, but not for the cardinal orientations

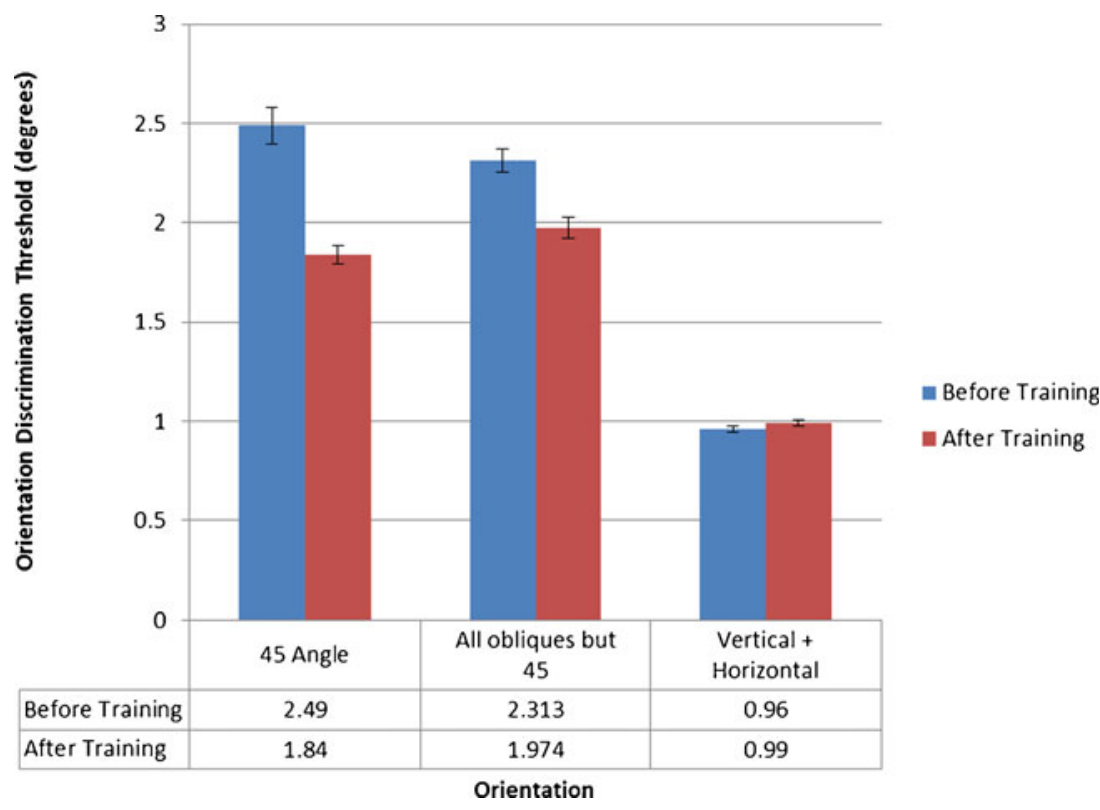

\section{Results}

Figure 2 shows the average orientation discrimination for the five observers in each of the eight meridians and for each asynchrony, before and after training in the 45-deg meridian, with the standard errors of the means.

For a more detailed analysis, a before-and-after comparison is afforded by the data presented graphically in Fig. 3, and in tabular form in Table 1, showing separately the trained meridian, the untrained oblique meridians, and the cardinal meridians. In the trained, 45-deg meridian, orientation discrimination thresholds (in degrees) improved from $2.49 \pm$ 0.09 to $1.84 \pm 0.05$, a $26 \%$ improvement averaged across all delay conditions $(p<0.05)$. We observed partial transfer to the other obliques (15\% improvement, $p<$ $0.01)$, but none to the cardinal orientations $(p>0.1)$. This is reflected in the oblique effect, calculated as the oblique divided by the cardinal thresholds, shown in Table 2. Incidentally, training the 45-deg meridian conferred no special benefit on its orthogonal, 135-deg, meridian, despite both being diagonal.

As can be seen in Fig. 4, not all of the five observers improved equally; interestingly, the observer with the best

Table 1 Percentage improvement in performance as a result of training on 45-deg meridians

\begin{tabular}{lllll}
\hline Onset Asynchrony (ms) & 0 & 250 & 500 & 750 \\
\hline Cardinal & 0 & -7 & -9 & 8 \\
Untrained orientation & 17 & 25 & 13 & 8 \\
Trained orientation & 22 & 23 & 30 & 27 \\
\hline
\end{tabular}

initial performance in fact showed a small, nonsignificant decrement.

In addition, two questions raised by the increase in the oblique effect that occurs when the comparison and the test line are temporally decoupled were answered. The first of these relates to possible short-term memory. Redrawing the data in Fig. 2 to highlight the threshold increase as a function of asynchrony between the comparison offset and the test-line onset (Fig. 5) makes it clear that the improvements resulting from training are not accompanied by any change in the asynchrony effect. No trend is seen that might be interpreted as a more lasting availability of the oblique standard to act as a comparison, as a consequence of the many thousands of training trials with feedback.

It has been postulated (Westheimer, 2003) that the superiority of performance when test and comparison lines are shown simultaneously could be due to a difference signal between the neural activity in neighboring orientations, which requires that they be elaborated synchronously. This proposition was tested in two observers who did not participate in the training experiments. The

Table 2 Oblique effect

Synchronous

All Delays

Untrained Oblique Meridians

$\begin{array}{lll}\text { Before } & 1.62 & 2.65 \\ \text { After } & 1.37 & 2.34 \\ \text { Trained Oblique Meridian } & & \\ \text { Before } & 1.70 & 2.82 \\ \text { After } & 1.41 & 2.02\end{array}$


Fig. 4 "Before training" and "after training" orientation discrimination thresholds for the 45-deg meridian and simultaneous presentation, shown individually for the five observers. Interestingly, the performance by the initially best observer remained stagnant

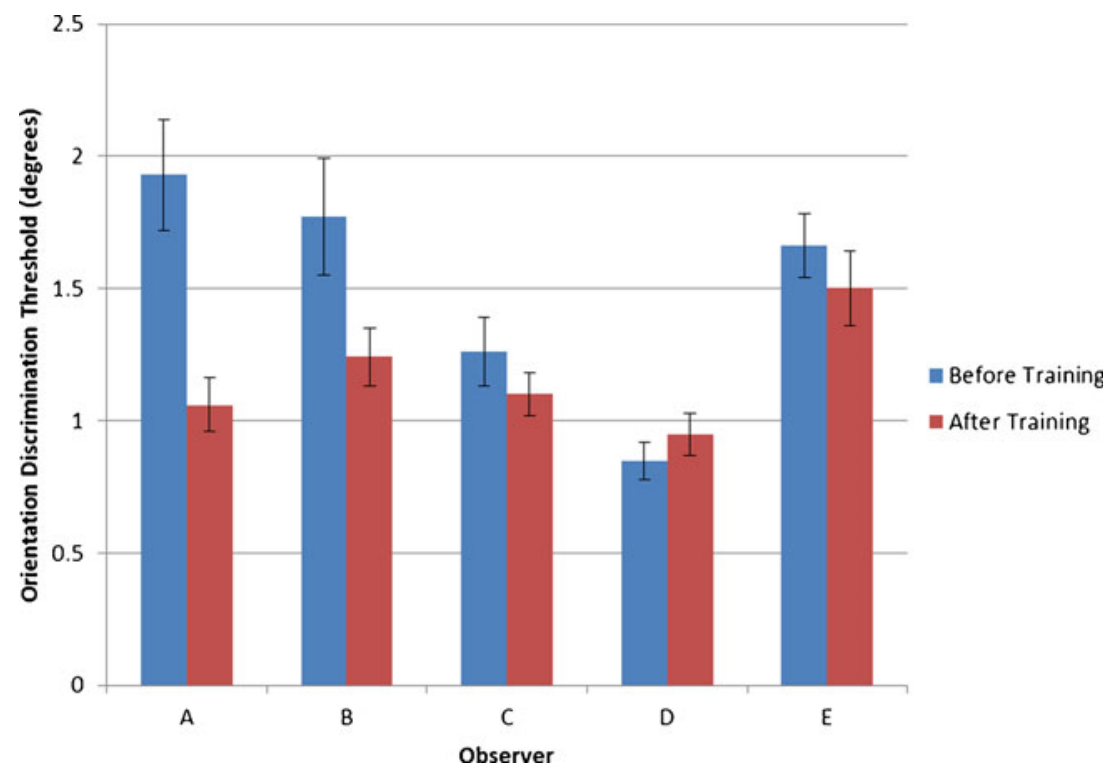

250-ms comparison flankers were replaced by a rectangle (see Fig. 6 top), which was either exposed continuously, blanked out for just the $250 \mathrm{~ms}$ during which the test line was shown, or blanked for an additional $400 \mathrm{~ms}$ preceding this. When the flanking stimulus was shown continuously (condition $\mathrm{C}$ ), the threshold was only insignificantly better than when the flanker was switched off during the test-line exposure (condition B). On the other hand, blanking out the comparison frame for an additional $400 \mathrm{~ms}$ preceding the stimulus onset (condition A) produced the threshold elevation typically seen with asynchrony ( $p<0.05$ in one observer, $p=0.1$ in the other).

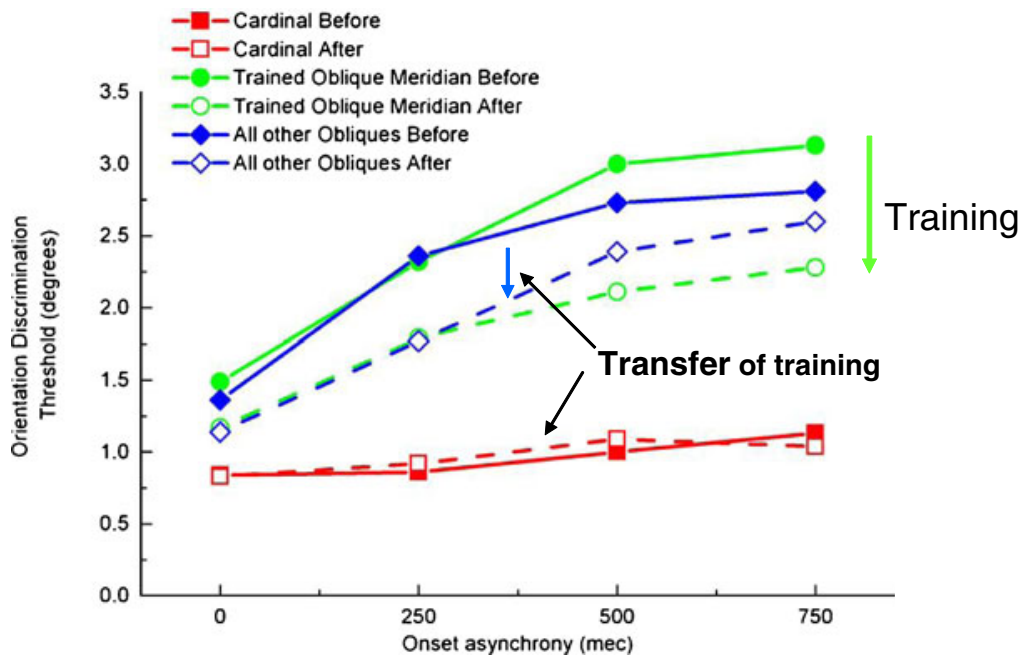

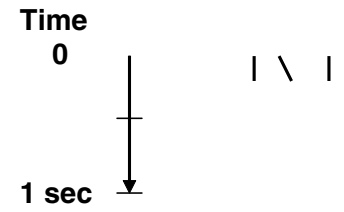

Fig. 5 Orientation discrimination thresholds for five observers as a function of the asynchrony between the comparison flankers and the test stimulus, for the initial control runs as well as for the posttraining evaluation. "Cardinal" curves represent the averages of horizontal and vertical data. "Untrained" data are the averages of the thresholds for all of the oblique meridians except the 45- deg meridian that was trained. The extent of transfer of training is indicated by the lengths of the arrows, which show improvements from training with the 45-deg meridian for performance on that meridian and on the other oblique meridians, and by the virtual superimposition of the "before" and "after" curves for the cardinal orientations 
Fig. 6 Orientation

discrimination thresholds for

two observers for a 45 -deg

oblique test line, shown for

$250 \mathrm{~ms}$, in the presence of a

comparison square that was

shown (a) continuously except

for the $400 \mathrm{~ms}$ preceding and

during the test line exposure,

(b) continuously except during

test-line exposure, or

(c) continuously
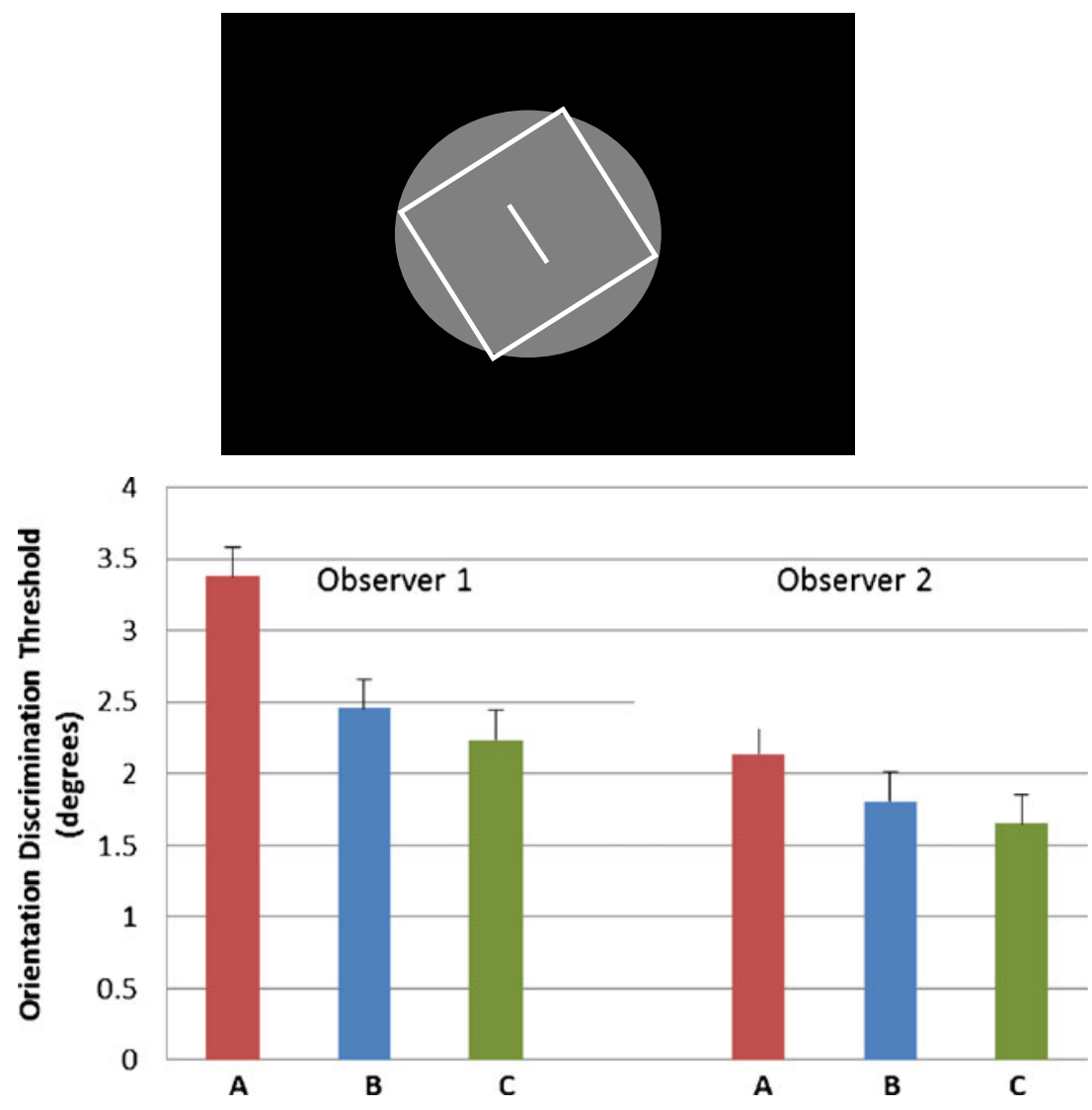

\section{Discussion}

Judging orientation or position?

When the orientation of a line is changed, so are the positions of its endpoints; hence, there is the possibility that the present experiment addresses the attribute of endpoint position and not of line orientation. Fortunately, when this question was asked explicitly in an earlier study (Westheimer, 1996), a very definite answer emerged: For foveal viewing of lines of the length used here, changes in orientation could be detected that are about three times smaller than the associated changes in the line ends when they are shown by themselves. In other words, connecting a point-pair improved by several times the discriminability of small relative displacements in the direction orthogonal to the line joining them. This finding underlies the proposition, basic to the present experiments, that line orientation is a primitive in spatial vision and that perceptual learning takes place in its domain.

\section{Perceptual learning}

Improved performance in a perceptual task with training, widely discussed under the heading of perceptual learning, is commonly regarded as the manifestation of synaptic facilitation in neural circuits that are active when the observer is engaged in the task. The range of orientations to which neurons in the primate primary visual cortex respond is relatively narrow. When perceptual learning has resulted in an improvement in the orientation discrimination for a short foveal line stimulus, it might be thought that this improvement would be restricted to the neural circuit composed of neurons whose orientation-tuning curve includes the trained orientation. The experiments reported here, however, reveal some improvement, though less, in other orientations as well. If the hyperacuity of orientation discrimination performance has its substrate in a local V1 circuit dedicated to and operating within a circumscribed range of orientations, the training transfer to removed orientations would have to be explained by a facilitating influence that is non-orientation-specific, and the results should be interpreted as demonstrating facilitation at two levels: first an orientation-specific level, presumably restricted to the circuit operating on the neurons directly affected by the training stimulus, and then another, more global level utilized in processing all orientations, probably farther along the neural-processing chain. This interpretation, however, is not sufficiently nuanced, because no improvement was observed in the vertical and horizontal, which were actually closer to the trained orientations than some of the meridians that did show improvement. Hence, fall-off that is proportional to the distance along the orientation dimension is not the reason. An economical conjecture for the robustness 
to performance enhancement of the cardinal meridians would be not so much the lack of a global, non-orientation-specific facilitating influence, but rather that they had already reached maximal performance. And indeed, the precision of less than a degree of arc in judging the orientation setting of a line $0.8 \mathrm{deg}$ in length falls into the hyperacuity category, once it is realized how small a fraction this is of the tuning width of the orientationselective cortical units through which the stimuli had been filtered. Some support for this proposition can be seen in the failure to manifest improvement of observer $\mathrm{D}$ in Fig. 4, whose initial threshold was lower than all of the other final thresholds.

\section{Cardinal orientations}

Our data do not speak to the question of whether the formidable threshold manifested by all of our observers in their initial pretraining tests had been reached as a result of long-term consistent exposure to horizontal and vertical contours in our "carpentered" environment, or whether the processing of contours in these meridians is inherently superior. Actually, given head and eye movements and perspective distortions, the putative horizontal/vertical predominance in visual scenes only rarely ends up being preserved on equivalent retinal meridians.

An alternative interpretation would be one that asserts an inherent dominance in the neural structure and organization subserving vertical and horizontal processing. In alert monkeys, the cortical orientation-selective neurons tuned to the horizontal and vertical are said to be more numerous than those attuned to obliques (Zhang et al., 2010), and an oblique effect has been reported in fMRI recordings from human V1 (Nasr \& Tootell, 2012). However, an immediate link between these three probes of visual response- $\mathrm{V} 1$ single-neuron orientation selectivity in the alert monkey, fMRI readouts from human V1, and performance in human psychophysical trials - is by no means a given. Orientation discrimination for visual stimuli that do not address such units explicitly, such as patterns made of vertical and horizontal contours merely implying an oblique orientation, also show such a deficit. Prominent oblique/cardinal anisotropies also occur in geometrical optical illusions (Weintraub, Krantz, \& Olson, 1980).

Simultaneous versus successive comparisons

The original impetus of this experiment was the question of whether the poorer performance on obliques when comparisons are temporally decoupled from a test stimulus - which can be viewed as short-term memory decay-might be mitigated by, or perhaps disappear with, training. One way to regard training is that it establishes a more robust internal representation of obliques, akin to the ones that seem to be available for cardinal directions at the outset.

Inspection of Fig. 5 suggests that this is not the case. Training improvement is essentially evenly distributed across all delays, including simultaneity, a finding that does not favor short-term memory enhancement through training.

This leaves the possibility that the greater oblique effect with delay (by virtue of attenuated availability of the comparison) might actually be a smaller oblique effect in simultaneity, due to a special orientation-differential detection apparatus, perhaps in the form of some short-term synaptic interaction in the primary visual cortex that fails when the two components are not synchronous. This proposition is made unlikely by the fact that the simultaneous-versussuccessive difference in oblique thresholds is almost obliterated (see conditions B and C in Fig. 6) when the otherwise continuous presence of an oblique square provides a strong perceptual frame, suggesting a more cognitive origin of the phenomenon.

Still, whatever role is finally assigned to a cognitive frame in the precision of judgments of the orientation of oblique contours, very substantial training-about 8,500 responses with feedback - in that task does not enable performance to reach the high level that the average observer manifests even on first viewing of contours in a cardinal orientation.

\section{References}

Appelle, S. (1972). Perception and discrimination as a function of stimulus orientation. Psychological Bulletin, 78, 266-278.

Heeley, D. W., \& Buchanan-Smith, H. M. (1992). Orientation acuity estimated with simultaneous and successive procedures. Spatial Vision, 6, 1-10.

McKee, S. P., \& Westheimer, G. (1978). Improvement in vernier acuity with practice. Perception \& Psychophysics, 24, 258-262.

Nasr, S., \& Tootell, R. B. (2012). A cardinal orientation bias in sceneselective visual cortex. Journal of Neuroscience, 32, 1492114926.

Raiguel, S., Vogels, R., Mysore, S. G., \& Orban, G. A. (2006). Learning to see the difference specifically alters the most informative V4 neurons. Journal of Neuroscience, 14, 6589-8602.

Schoups, A. A., Vogels, R., \& Orban, G. A. (1995). Human perceptual learning in identifying the oblique orientation: Retinotopy, orientation specificity and monocularity. The Journal of Physiology, 483, 797-810.

Shiu, L.-P., \& Pashler, H. (1992). Improvement in line orientation discrimination is retinally local but dependent on cognitive set. Perception \& Psychophysics, 52, 582-588.

Tschopp-Junker, C., Gentaz, E., \& Viviani, P. (2010). Effect of selective and distributed training on visual identification of orientation. Experimental Brain Research, 202, 605-611. doi:10.1007/ s00221-010-2167-9 
Vogels, R., \& Orban, G. A. (1985). The effect of practice on the oblique effect in line orientation judgments. Vision Research, 25, 1679-1685.

Weintraub, D. J., Krantz, D. H., \& Olson, T. (1980). The Poggendorff illusion: Consider all the angles. Journal of Experimental Psychology. Human Perception and Performance, 6, 718-725.

Westheimer, G. (1996). Location and line orientation as distinguishable primitives in spatial vision. Proceedings of the Royal Society B, 263, 503-508.
Westheimer, G. (2003). Meridional anisotropy in visual processing: Implications for the neural site of the oblique effect. Vision Research, 43, 2281-2289.

Zhang, E., Huang, M., Xiang, X., Yan, Y., Shen, Y., Chen, M., ... Li, W. (2010). Anisotropic orientation selectivity of monkey V1 neurons in Cartesian and polar coordinates (Poster No. 483.12/ UU2). 2010 Neuroscience Meeting Planner. San Diego, CA: Society for Neuroscience. 